\title{
MASAS MESENTERICAS: EVALUACION POR TOMOGRAFIA COMPUTADA
}

\author{
Int. Francisco Riquelme $M^{(1)}$, Drs. Luis Vega $P^{(2)}$, Andrés $O^{\prime} B r i e n S^{(3)}$.
}

1. Interno de Medicina, Facultad de Medicina. Pontificia Universidad Católica de Chile.

2. Residente de Radiología, Departamento de Radiología. Hospital Clínico Pontificia Universidad Católica de Chile.

3. Departamento de Radiología, Hospital Clínico Pontificia Universidad Católica de Chile.

\section{MESENTERIC MASSES. COMPUTED TOMOGRAPHY ASSESSMENT}

\begin{abstract}
The mesentery is a frequent site for abdominal diseases, since it constitutes the intestinal scaffolding and pathway for many structures. Nowadays, computed tomography $(C T)$ is the imaging modality of choice for evaluating mesenteric masses and their origin. An isolated mesenteric mass (primary) is an unexpected and rare tomographic finding in the context of patients with nonspecific clinical signs. The identification of a primary mesenteric mass requires an effort to exclude the possibility of being a secondary source. Thus, knowledge of the spectrum of mesenteric disease (neoplastic, infectious, inflammatory, vascular, traumatic, congenital) and its computed tomographic appearance is the key for proper diagnosis and treatment.

Keywords: mesenteric masses, mesentery, computed tomography (CT).
\end{abstract}

Resumen: El mesenterio es un sitio frecuente de enfermedades abdominales, ya que constituye el soporte intestinal y una vía de paso para muchas estructuras. Hoy en día, la tomografía computada (TC) es la modalidad de imagen de elección para evaluar las masas mesentéricas y su origen. Una masa aislada (primaria) es un hallazgo tomográfico raro e inesperado de encontrar en el contexto de pacientes con signos clínicos inespecíficos. La identificación de una masa mesentérica primaria requiere un esfuerzo para descartar la posibilidad de un origen secundario. Así pues, el conocimiento del espectro de la enfermedad mesentérica (neoplásicas, infecciosas, inflamatorias, vasculares, traumáticas y congénitas procesos) y su apariencia tomográfica es clave para el diagnóstico y el tratamiento adecuado.

Riquelme F y cols. Masas mesentéricas: Evaluación por tomografia computada. Rev Chil Radiol 2009; 15: 165-173.

Correspondencia: Dr. Andrés O'brien S.

obrienandres@yahoo.com

Trabajo recibido el 22 de julio de 2009, aceptado para publicación el 10 de noviembre 2009.
Palabras clave: Masas mesentéricas, Mesenterio, Tomografía computada.

\section{Introducción}

La cavidad peritoneal se encuentra recubierta por una capa de tejido conectivo y células epiteliales, formando una capa visceral y parietal, que se refleja sobre sí misma formando el mesenterio. Este fija el yeyuno e ileon, junto a sus estructuras vasculares, neurales, linfáticas y tejidos mesenquimáticos a la pared abdominal posterior. Su raíz se extiende por quince centímetros oblicuamente de izquierda a derecha, desde el ángulo duodenoyeyunal hasta la región ileocecal. De esta manera, la cavidad abdominal queda divida en distintos espacios virtuales.

Por su relación con las estructuras descritas, el mesenterio es asiento frecuente de procesos neoplásicos, primarios o secundarios, quistes y otras lesiones. La clínica es inespecífica, y en la mayoría de los casos estará determinada por la causa de origen (generalmente neoplasias de otros territorios) o sus complicaciones. Se reporta dolor abdominal, baja de peso, diarrea, masa palpable y sangre en deposiciones como los más frecuentes, siendo la TC la modalidad de elección para su diagnóstico.

Los tumores secundarios, mucho más frecuentes, llegan al mesenterio por distintas vías de diseminación: directa desde el intestino delgado, linfática, hematógena o siembra peritoneal. Otras causas, como las inflamatorias o infecciosas, se mimetizan con facilidad con las causas neoplásicas (tuberculosis, adenitis, fibromatosis) y deben ser consideradas según el contexto clínico.

A continuación revisaremos los aspectos relacionados con el diagnóstico de masas mesentéricas a través de TC, con énfasis en las de origen neoplásico (primarias y secundarias), así como también en su diagnóstico diferencial.

\section{Masas neoplásicas primarias}

Las neoplasias primarias del mesenterio son infrecuentes. Derivan en su mayoría del mesénquima y generalmente tienen un comportamiento benigno ${ }^{(1,2)}$. 
Los más frecuentes son el tumor desmoides y el lipoma. Es posible encontrar además leiomiosarcomas, fibrosarcomas, schwannomas, linfoma y GIST primarios (gastrointestinal stromal tumor), cuyo conocimiento deriva fundamentalmente del reporte de casos.

\section{Tumor desmoides}

La palabra desmoide deriva del griego "desmos" que significa banda o tendón. Esta es una lesión fibroblástica localmente agresiva, que tiende a recurrir localmente sin metástasis a distancia. Estos tumores son considerados de malignidad intermedia. Típicamente afectan la pared abdominal (50\%), mesenterio $(41 \%)$ y retroperitoneo (9\%). De aparición esporádica, se ha atribuido su origen a cirugía y/o trauma previos, factores inflamatorios sistémicos, hormonales ${ }^{(2)}$ (hiperestrogenismo, embarazo) y genéticos ${ }^{(2-4)}$ (enfermedad de Gardner o poliposis adenomatosa familiar). El tumor desmoides intraabdominal afecta mayormente a mujeres, con uno o más hijos, entre la quinta y sexta década. Es asintomático en la mayoría de los casos, pero ha sido documentado dolor abdominal leve sin masa palpable, así como sus complicaciones agudas $^{(5)}$. Su tratamiento es quirúrgico, con tasas de recurrencias que llegan al $65 \% \%^{(1,3,6)}$.

En la TC se observan lesiones generalmente únicas (13-28\% múltiples), sólidas, con densidad mayor o igual a la del músculo (Figura 1), cuyos márgenes pueden estar bien o regularmente definidos cuando se localizan en la pared abdominal ${ }^{(2,3)}$. No poseen un patrón de refuerzo característico: $12 \%$ son hipodensos, $41 \%$ hiperdensos y $47 \%$ isodensos. Su apariencia es homogénea en el $71 \%$, con tamaños que varían entre $1 \mathrm{a} 30 \mathrm{~cm}$ con una media de $8 \mathrm{~cm}$. A diferencia de aquellos ubicados en la pared abdominal, la mayoría de los tumores desmoides mesentéricos no tienen bordes bien definidos ( $41 \%$ bien definidos, $24 \%$ patrón infiltrativo y $35 \%$ mixtos $)^{(2,7)}$. Pueden generar complicaciones intestinales agudas, situación que ha sido reportada con más frecuencia en pacientes

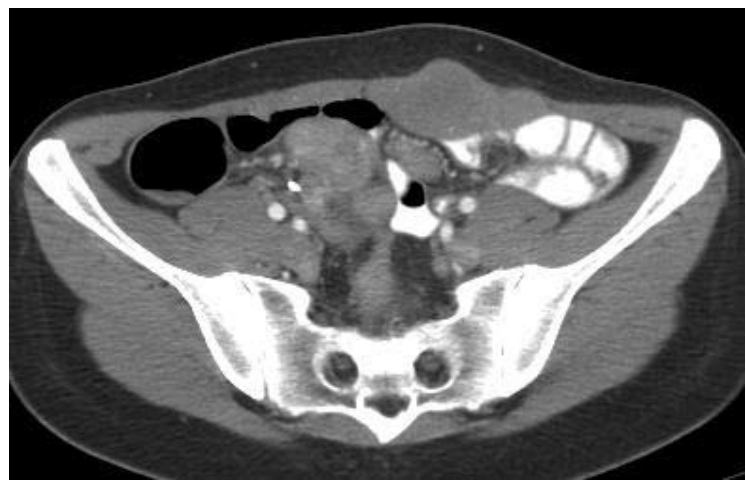

Figura 1. Tumor desmoides mesentérico y de pared abdominal. TC corte axial, se observan masas que refuerzan con contraste a nivel de pared abdominal y mesenterio. colectomizados por síndrome de Gardner, con hasta un $32 \%$ de hidronefrosis y $20 \%$ de obstrucción intestinal secundarios a fibromatosis mesentérica. Dentro del diagnóstico diferencial se debe considerar linfoma ${ }^{(8,9)}$, tumor carcinoide y mesenteritis esclerosante ${ }^{(5)}$.

\section{EGIST mesentérico}

EI GIST constituye el grupo más frecuente de neoplasias derivadas del mesénquima en el tracto digestivo. Ocurren con igual frecuencia en hombres y mujeres, mayores de 50 años, con 60 a $70 \%$ de ubicación gástrica, 20 a $30 \%$ de intestino delgado, $10 \%$ colorrectal y $<$ de $5 \%$ esofágico ${ }^{(10-12)}$ (único segmento en que predominan los leiomiomas por sobre los GIST). Difieren de los verdaderos leiomiomas, leiomiosarcomas y shwannomas desde un punto de vista histológico, inmunohistoquímico y genético. Derivan de las células intersticiales de Cajal y están definidos por la expresión de KIT (CD117), un receptor transmembrana expresado normalmente en células stem. Aún cuando es habitual el compromiso secundario de mesenterio y omento por GIST intestinales, en estas dos localizaciones se presentan las formas de GIST primario extradigestivo más frecuentes (EGIST) ${ }^{(11,13)}$. A diferencia de los GIST que en un 70 a $80 \%$ son benignos, y como en el resto de las ubicaciones extras gástricas, los EGIST de mesenterio son predominantemente malignos, encontrándose enfermedad metastásica hasta en un $50 \%$ de los casos $^{(14)}$. Los factores determinantes de malignidad son: ubicación, tamaño, características histológicas (índice mitótico) y presencia de enfermedad metastásica (hepática y peritoneal; el compromiso nodal es infrecuente) ${ }^{(14,15)}$. El perfil definitivo de los EGIST está por definirse aún, existiendo reportes hasta la fecha que muestran un patrón genético, inmunohistoquímico y clínico comparable con sus semejantes digestivos ${ }^{(13,16)}$. En la TC son tumores bien definidos, de aspecto heterogéneo, de contornos lobulados, alcanzando tamaños promedios mayores que los intestinales $(12-16,5 \mathrm{~cm})^{(13,15-17)}$, lo que se explica por la escasa e inespecífica sintomatología asociada. Estas masas presentan un componente sólido periférico correspondiente a tumor viable, que refuerza con el medio de contraste ${ }^{(18)}$. Las áreas de baja atenuación central determinadas por necrosis, hemorragia y degeneración quística se asocian a malignidad ${ }^{(16)}$. A menudo, los EGIST mesentéricos y omentales son tan grandes que resultan inseparables de la pared intestinal, siendo difícil diferenciar su origen en la TC, a menos que contengan zonas de ulceración, cavidades 0 aire que orienten a GIST intestinales ${ }^{(15,18)}$. EI tumor desmoides y otros pseudotumores inflamatorios son bien delimitados, con patrones de atenuación homogéneos y constituyen las primeras alternativas diagnósticas diferenciales al EGIST mesentérico, siempre que no haya evidencias de hemorragia, 
necrosis o degeneración quística. La existencia de adenopatías hará pensar en linfoma. Como todo GIST, su apariencia podría resultar indistinguible de otros sarcomas, leiomiosarcomas, histiocitoma maligno, fibrosarcoma o liposarcoma, aunque estos suelen tener una apariencia más invasiva.

\section{Linfoma mesentérico}

El linfoma primario mesentérico ha sido reportado en escasas series. La más importante informa 11 casos sobre un total de 92 pacientes con linfoma primario extranodal intestinal. Como en otros reportes, destaca que su tipo histológico y comportamiento es más cercano al de linfomas nodales (linfoma folicular) que intestinales ${ }^{(19)}$. El aspecto tomográfico es semejante al descrito en linfomas que infiltran secundariamente el mesenterio.

\section{Masas neoplásicas secundarias}

Se han descrito distintas vía de invasión mesentérica. Por extensión directa lo hace el carcinoide y otros tumores intraabdominales como el de colon y páncreas (Figura 2). Por siembra peritoneal se diseminan el cáncer de estómago, intestino delgado, colon, páncreas, y menos frecuentemente el ovárico. Por diseminación linfática se siembran linfoma y algunas neoplasias epiteliales. La embolía metastásica del mesenterio está descrita en cáncer de mama, pulmón y melanoma.
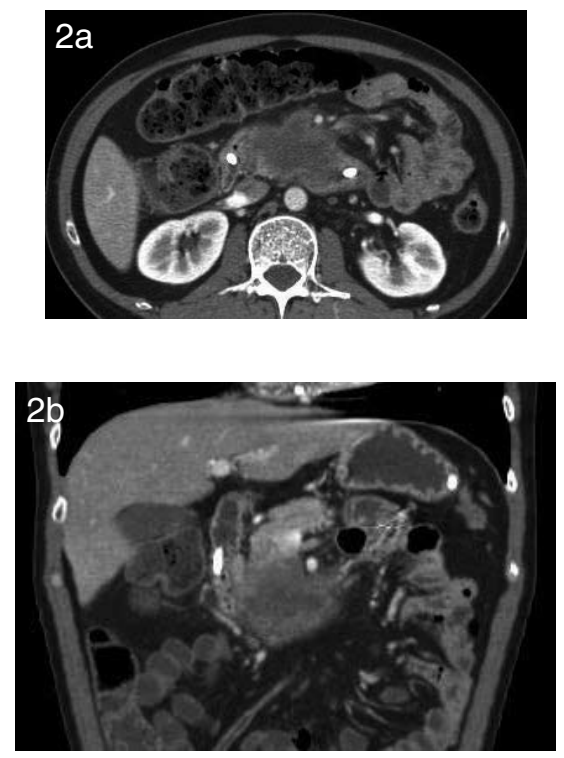

Figura 2 a y b. Infiltración mesentérica por cáncer de páncreas (proceso uncinado). Corte axial y reformateo coronal de TC. Se identifica una masa infiltrante, dependiente del proceso uncinado del páncreas.

\section{Tumor carcinoide gastrointestinal}

Los tumores carcinoides, derivados del sistema endocrino difuso, en su mayoría ocurren en intestino delgado o apéndice, donde representan el 6 a 35\% de las neoplasias primarias ${ }^{(20-23)}$. Aunque considerados malignos, su comportamiento es variable, aún en presencia de metástasis. Los tumores primarios son usualmente submucosos y pequeños $\left(<2 \mathrm{~cm}^{(24)}\right.$, preferentemente de ileon distal $(90 \%)^{(22,23)}$ y se pueden localizar incidentalmente, como por ejemplo en la enteroclisis por TC ${ }^{(25)}$. Pueden visualizarse como nódulos o masas bien definidas, hipervascularizadas o simplemente como un engrosamiento de la pared intestinal ${ }^{(26)}$. Se presentan en la sexta década de la vida, sin diferencia de género. Su diagnóstico es tardío e incidental en más de la mitad de los casos (obstrucción intestinal o biopsia de apendicetomía ${ }^{(27-30)}$, debido a la inespecificidad de los síntomas (dolor abdominal, sangre en deposiciones, síndrome carcinoide). Entre el 40 a $80 \%$ comprometen el mesenterio, ya sea por extensión directa o linfática ${ }^{(3,27,31)}$. Las localizaciones secundarias se detectan con mayor facilidad, siendo en general hipervascularizadas y de bordes espiculados, por la reacción desmoplástica (Figura 3 ); éstas pueden estar en estrecho contacto con la lesión primaria (Figura 4). La reacción desmoplástica descrita puede determinar tracción, con angulación y obstrucción de asas adyacentes. Esto es resultado de una reacción de fibrosis retráctil en que se produce fijación y estrechamiento luminal por engrosamiento parietal y mesentérico ${ }^{(27,29,32)}$. La infiltración tumoral, así como la isquemia secundaria son implicadas como causas $^{(28)}$. La presencia de calcificaciones es muy sugerente, visible hasta en el $70 \%$ de los $\operatorname{casos}^{(24,31)}$. Las arterias mesentéricas muestran un curso tortuoso por la esclerosis vascular elástica así como por las estenosis multifocales en la periferia de éstas. La isquemia mesentérica secundaria es una causa de complicación de estos tumores. El compromiso nodal regional y retroperitoneal de los tumores carcinoides ocurre en un 50 a $85 \%{ }^{(29)}$, siendo esto evidente en la TC hasta en el $35 \%$ de los casos. Puede asociarse también a ascitis y metástasis hepáticas, hasta en un $65 \%{ }^{(27)}$; las metástasis hepáticas son hipervasculares $^{(28)}$.

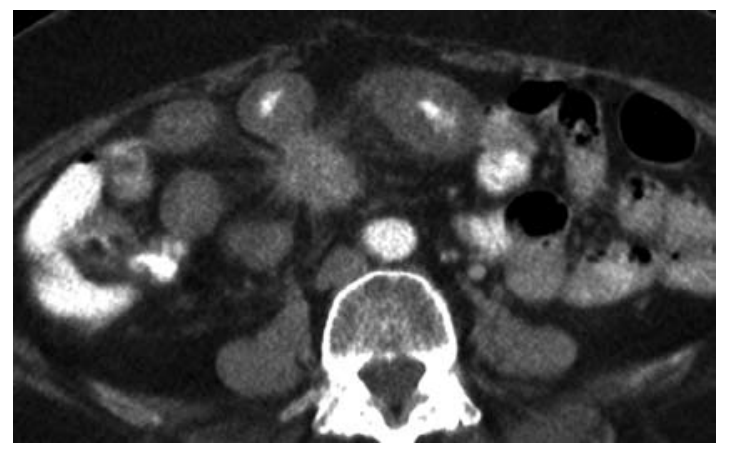

Figura 3. Carcinoide metastásico. Corte axial de TC, se identifica una masa mesentérica espiculada asociada a reacción desmoplástica. 


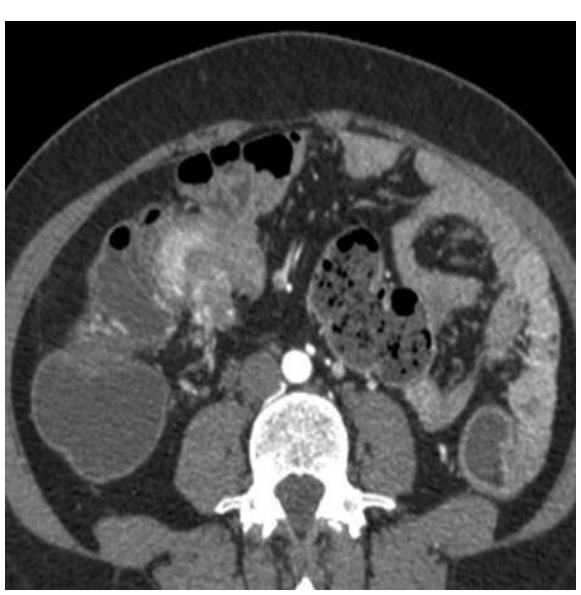

Figura 4. Carcinoide ileal con compromiso mesentérico. Corte axial de TC muestra una masa hipervascular en íleon, en estrecho contacto con una masa mesentérica.

En el diagnóstico diferencial debe considerarse enfermedad de Crohn, mesotelioma peritoneal, enfermedad metastásica de otro origen (ovario o colon) e incluso linfoma. Además, tumores submucosos carcinoides pequeños son similares al GIST, si bien éstos no se asocian a presencia de adenopatías.

\section{Linfoma}

El linfoma no Hodgkin (LNH) representa entre el 25 y el $40 \%{ }^{(33-35)}$ de las neoplasias de intestino delgado, correspondiendo a la ubicación extranodal más frecuente $(20-40 \% \text { de los } \mathrm{LNH})^{(33,35-37)}$. Ya sea por enfermedad primaria gastrointestinal o por localización secundaria, el $\mathrm{LNH}$ presenta compromiso mesentérico hasta en el $51 \%$ de los $\operatorname{casos}^{(38,39)}$, constituyendo la primera causa de masas mesentéricas neoplásicas ${ }^{(5)}$. El linfoma no Hodgkin intestinal se asocia a pacientes inmunocomprometidos (VIH), enfermedad celíaca, Crohn, lupus y quimioterapia. Mayoritariamente afecta la región ileal distal, con grandes lesiones de más de $10 \mathrm{~cm}^{(34,40)}$. La presentación clínica es inespecífica al igual que en el resto de las neoplasias primarias del intestino delgado, predominando: dolor abdominal, nauseas, vómitos y pérdida de peso hasta en un tercio de los casos; se observa fiebre en menos del $5 \%{ }^{(36)}$. El compromiso intestinal por LNH produce engrosamiento parietal anular, lesiones excavadas (masas tumorales ulceradas) y/o compromiso mesentérico aislado. En la TC, característicamente se observan múltiples masas homogéneas redondeadas (adenopatías) con densidad de partes blandas que envuelve vasos y grasa mesentérica, formando el "signo del sandwich" (Figura 5). Con la administración de contraste endovenoso, las estructuras mesentéricas tubulares se contrastan, resaltando la apariencia del "relleno del sandwich" $(9,41,42)$. Se describen casos en los que en relación al crecimiento de las adenopatías se genera obstrucción parcial del retorno venoso, con aumento del diámetro de las venas. Se pueden observar además masas de gran diámetro, que desplazan asas intestinales y poseen un patrón determinado por áreas de necrosis. Frecuentemente, extensas adenopatías retroperitoneales acompañan el compromiso mesentérico, siendo en muchos casos la clave del diagnóstico ${ }^{(43)}$.

El diagnóstico diferencial debe hacerse con enfermedades inflamatorias (infección por micobacterias, enfermedades inflamatorias y vasculares).

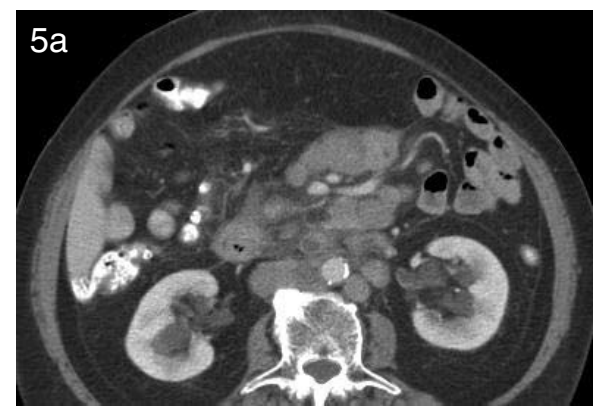

Figura 5 a y b. Linfoma con compromiso mesentérico. Corte axial y reformateo sagital de TC, muestra múltiples adenopatías que rodean los vasos mesentéricos.

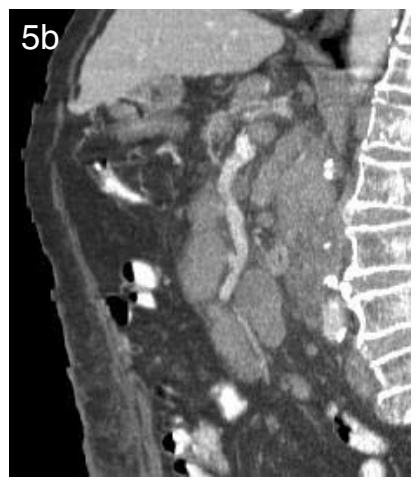

\section{Quistes mesentéricos}

Los quistes mesentéricos y omentales son lesiones infrecuentes, existiendo pocas series radiográficas reportadas ${ }^{(44,45)}$. En este grupo se incluyen lesiones de distinto origen y significado patológico, en su mayoría benignas. Al menos un tercio de los casos afectan a pacientes menores de 10 años ${ }^{(45)}$, siendo considerados en su mayoría congénitos, por sobre otras causas como postraumáticas, infecciosas o neoplásicas ${ }^{(45)}$. Se extienden por todo el tracto intestinal, concentrándose en ileon distal ${ }^{(5)}$. Un $80 \%$ de los casos presenta síntomas, entre los que destaca la masa palpable (30-50\%). Con menor frecuencia son causa de complicaciones agudas como obstrucción intestinal, hemorragia abdominal, vólvulo intestinal o torsión del quiste ${ }^{(5,46,47)}$. Si bien la ecografía abdominal puede orientar al diagnóstico en un inicio, la TC es capaz de entregar información más concluyente. El diagnóstico diferencial debe realizarse con otras lesiones quísticas abdomino-pelvianas (ováricas, 
del uraco, riñones poliquísticos, pseudoquistes de páncreas). El quiste mesentérico más frecuente es el linfangioma, seguido por el pseudoquiste no pancreático, quiste de duplicación intestinal, quiste entérico y teratoma, entre otros.

\section{Linfangioma}

Es una malformación linfática congénita sin comunicación con el sistema linfático intestinal(45,48). Se ha registrado sólo en niños y adolescentes (edad promedio: 16 años) ${ }^{(49)}$, todos sintomáticos (distensión y dolor abdominal, masa palpable, vómitos y diarrea). Por lo general, se trata de grandes masas que también pueden localizarse en el omento, llegando a medir hasta $40 \mathrm{~cm}$ de diámetro mayor, generando desplazamiento de asas intestinales y pseudoobstrucción ${ }^{(44)}$. En la TC se visualizan lesiones de pared fina, multiloculadas, con contenido de baja atenuación (seroso/sero-hemorrágico), que en algunos casos llega a densidad grasa (quiloso) ${ }^{(44,48)}$. En forma variable se observa refuerzo de los septos y calcificaciones aisladas.

\section{Otras lesiones quísticas}

Entre los diagnósticos diferenciales se debe considerar el quiste de duplicación intestinal, anomalía de origen congénito que se origina en cualquier porción del tracto gastrointestinal, de preferencia ileon distal ${ }^{(50-52)}$. Son lesiones quísticas únicas $(82 \%)^{(51)}$, uniloculares, que alcanzan un tamaño de hasta 15 cm según algunas series, siendo intra o extraluminales ${ }^{(44,50)}$. Están delimitados por pared intestinal normal incluyendo mucosa heterotópica (generalmente gástrica o pancreática) y por lo general poseen contenido de baja atenuación (seroso) ${ }^{(48-51)}$.

Similares características posee el quiste intestinal, que a diferencia de los de duplicación, deriva de divertículos gástricos o colónicos que migran al mesenterio, formando quistes uniloculares de paredes finas (mucosa con componente fibroso de pared intestinal, sin capa muscular). En la TC se observa un quiste único, con contenido de baja atenuación (seroso), de paredes imperceptibles. Su aspecto en la TC es indistinguible de un quiste mesotelial.

El quiste mesotelial es una neoplasia benigna originada de células serosas, que puede encontrarse en el espacio pleural, pericárdico y peritoneal. Su localización mesentérica es la menos frecuente y, a diferencia de los mesoteliomas malignos, no se relacionan con exposición a asbesto ni dan metástasis a distancia, siendo localmente recurrentes, con mayor frecuencia entre mujeres jóvenes menores de 40 años. Su origen se cree secundario a inflamación y/o trauma. Habitualmente son extensos quistes multiloculados con paredes imperceptibles y contenido seroso. El análisis anatomopatológico informa una superficie interna recubierta por células mesoteliales ${ }^{(48)}$. Debe considerarse además el pseudoquiste no pancreático, cuyo origen se cree secundario a la degeneración de hematomas o abscesos mesentéricos ${ }^{(44)}$, sin relación con pancreatitis. No poseen capa interna celular y en ocasiones muestran contenido hemorrágico o purulento. En la TC poseen una pared engrosada y es frecuente la presencia de niveles líquido-líquido en su interior.

\section{Teratoma o tumor dermoides}

Son neoplasias derivadas de células pluripotenciales que presentan tejidos relativamente especializados, de las tres capas germinales. Su localización más habitual es gonadal en adultos, mientras en niños es la sacrococcígea. Su ubicación gastrointestinal es infrecuente, por lo general en línea media o parasagital, siendo la mayoría benignos $(85 \%)^{(53,54)}$. Es habitual un acercamiento inicial con una radiografía de abdomen simple que muestra una masa de partes blandas, con calcificaciones hasta en un 35 a $60 \%$ de los casos $^{(53)}$. En la TC se observan lesiones por lo general únicas, redondeadas y capsuladas, con uno o más componentes quísticos (hipodensas) que pueden constituir hasta el $50 \%$ de la lesión. Se identifican además áreas sólidas, con intensidad de partes blandas o grasa, esto último característicamente en la periferia. Calcificaciones pequeñas de bordes irregulares están presentes en la mayoría de los casos y son el pilar del diagnóstico, junto a la presencia de contenido graso (Figura 6). El principal desafío es distinguir si verdaderamente se trata de un teratoma mesentérico o un gran teratoma pélvico, mucho más frecuente ${ }^{(5,53,54)}$.

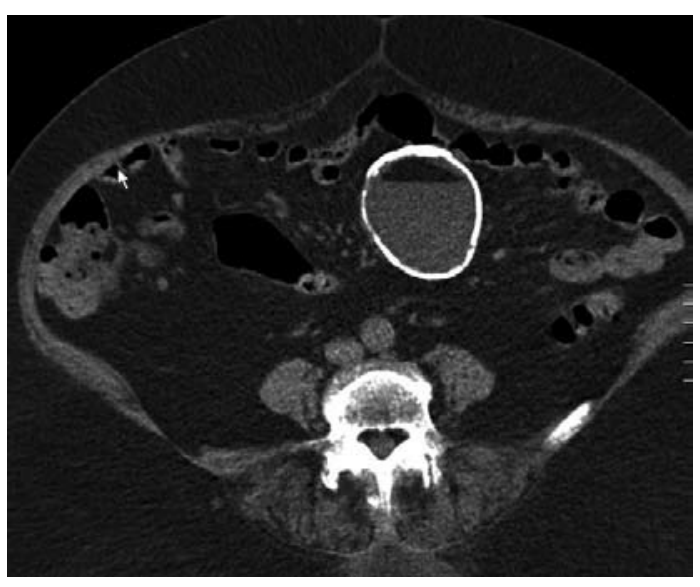

Figura 6. Quiste dermoides o teratoma mesentérico. Corte axial de TC, muestra una masa mesentérica quística compleja, con calcificaciones periféricas y contenido graso.

\section{Procesos mesentéricos inflamatorio-infecciosos TBC abdominal y compromiso mesentérico}

Distintos reportes muestran aumento en las tasas de TBC, en particular de cepas multiresistentes, incluso en países desarrollados concentrándose en 
pacientes inmunocomprometidos ( $\mathrm{VIH}$ o tratamiento corticoidal crónico), alcohólicos y usuarios de drogas endovenosas ${ }^{(55-57)}$. La ubicación gastrointestinal es la más común de las extrapulmonares, con preferencia por vísceras sólidas. Considerando que en la población referida existe con más frecuencia TBC extrapulmonar (hasta en un 30\%)(55), la TBC abdominal debe incluirse en los diagnósticos diferenciales de compromiso mesentérico. Su presentación clínica es inespecífica; el estudio con TC revela en casi todos los casos linfadenopatías mesentéricas y peripancreáticas, con afectación simultánea de múltiples grupos que característicamente presentan nódulos con refuerzo periférico e hipoatenuación central (Figura 7 ), indicativo de necrosis de caseificación ${ }^{(58,59)}$. Este hallazgo obliga a pensar también en enfermedad de Whipple ${ }^{(60)}$, infección piogénica, localización secundaria por metástasis, y en pacientes inmunocomprometidos, linfoma, sarcoma de Kaposi e infección por micobacterias atípicas (MAI) ${ }^{(61,62)}$. Pueden verse además conglomerados nodales con distintos patrones de atenuación: homogéneos, mixtos y también normales ${ }^{(58)}$. El compromiso linfático con predominio de mesenterio y omento, respecto a retroperitoneo, es muy sugerente de TBC intestinal, al igual que lo es la presencia de fístulas ${ }^{(62)}$. Pese a que pueden a llegar a generar grandes conglomerados de adenopatías, no se asocian a complicaciones obstructivas (biliar, vías urinarias o intestinal), por lo que su presencia debe plantear un origen distinto a TBC. Otros elementos extramesentéricos que aportan al diagnóstico son: hallazgo frecuente de esplenomegalia $(70 \%)$ y peritonitis "húmeda" (30\%) que traduce enfermedad tuberculosa abdominal diseminada $(90 \%)^{(58-62)}$. Se observa ascitis libre o loculada, que usualmente presenta densidad levemente mayor a la del agua por alto contenido proteico y celular $(20-55 \mathrm{HU})^{(58,62)}$. El caso opuesto es la peritonitis "seca" en que hay engrosamiento del mesenterio, adhesiones fibrosas y nódulos de caseificación. Cuando adquiere un patrón irregular de engrosamiento peritoneal obliga a descartar carcinomatosis peritoneal(58). En resumen, los hallazgos no son patognomónicos y deben ser interpretados en el contexto del paciente, sin olvidar otras causas de peritonitis.

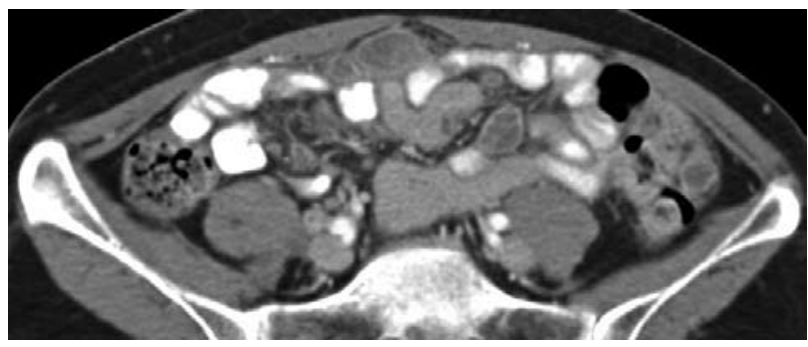

Figura 7. Tuberculosis ganglionar mesentérica. Corte axial de TC, muestra múltiples adenopatías mesentéricas con centro hipodenso.

\section{Paniculitis intraabdominal (o mesenteritis esclerosante)}

Es una enfermedad idiopática ${ }^{(63,64)}$, extremadamente inusual, en que ocurre un trastorno inflamatorio de la grasa intraabdominal (preferentemente mesentérica) que determina la formación de masas con focos de necrosis. Algunos autores diferencian el término paniculitis mesentérica de mesenteritis esclerosante, considerando el primero como una inflamación aguda inespecífica (primaria o secundaria a otras patologías). Cuando ésta ocurre en el marco de un proceso crónico, deriva en fibrosis mesentérica, lo que constituye la mesenteritis esclerosante ${ }^{(63)}$ (Figura 8). La sintomatología es vaga e inespecífica, siendo común el dolor abdominal asociado a fiebre, náuseas, vómitos y masa palpable. Por lo general, no produce compromiso de pared intestinal ni complicaciones agudas ${ }^{(65)}$. En la TC se observa desde un sutil incremento en la atenuación mesentérica a la aparición de masas mesentéricas de moderado tamaño (hasta $15 \mathrm{~cm}$ ), de paredes bien definidas, heterogéneas, con áreas de densidad cercana al agua, grasa y partes blandas. Estas últimas representan infiltrado inflamatorio, edema y fibrosis secundaria ${ }^{(64)}$. Poseen característicamente un centro necrótico. Esta masa puede generar estenosis por compresión extrínseca de vasos, con desarrollo de circulación colateral secundaria. Existe preservación de la grasa perivascular, lo que es descrito como el signo del "anillo graso", que junto con la presencia de calcificaciones ubicadas en centro necrótico descrito distinguen estas masas mesentéricas de otros procesos mesentéricos como el linfoma, tumor carcinoide y carcinomatosis ${ }^{(64,66)}$. El páncreas debe conservar su arquitectura para descartar que se trate de un proceso secundario a inflamación de éste. El diagnóstico diferencial debe incluir: tumor dermoides, del que se distingue por el contexto clínico y el carácter quístico de éste último, y liposarcomas, que generalmente son de gran tamaño, no capsulados, con áreas de densidad de partes blandas y otras de grasa macroscópica, sin centro necrótico. En muchos casos pueden ser indistinguibles ${ }^{(64)}$.

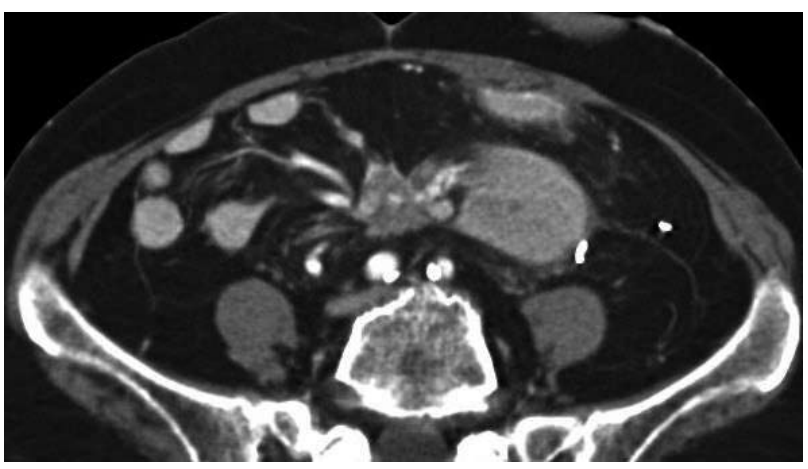

Figura 8. Mesenteritis esclerosante. Corte axial de TC: masa mesentérica que determina tracción y obstrucción de asas de intestino delgado. 


\section{Enfermedad de Crohn}

La enfermedad de Crohn compromete frecuentemente el mesenterio, identificándose formación de abscesos, flegmones y proliferación del tejido adiposo, en TC. Los cambios inflamatorios que determinan aumento de densidad de la grasa y órganos adyacentes generan borramiento de las estructuras, observándose pérdida de la interfase entre la pared intestinal y el mesenterio. El mesenterio está hipervascularizado, con dilatación de la vasa recta y distanciamiento de sus componentes, lo que se conoce como "signo de la peineta"(67). Usualmente, se identifican adenopatías mesentéricas de carácter reactivo, hallazgo que obliga a considerar la presencia de linfoma o carcinoma en el diagnóstico diferencial, que se dan frecuentemente en pacientes con Crohn ${ }^{(68)}$. Se completa el diagnóstico si se identifica un engrosamiento parietal con estratificación de la pared, como ocurre en el $80 \%$ de los $\operatorname{casos}^{(69,70)}$, hiperrefuerzo mucoso intestinal, tractos sinuosos, fístulas enteroentéricas, enterocólicas o enterocutáneas (Figura 9) y/o enfermedad perianal.

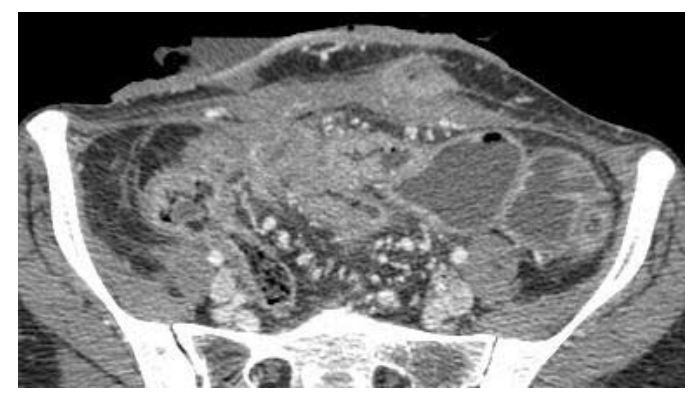

Figura 9. Enfermedad de Crohn severa con compromiso mesentérico. Corte axial de TC: se observa una masa inflamatoria mesentérica asociada a fístulas enteroentéricas y enterocutáneas.

\section{Otros}

\section{Leiomioma parasítico}

Los tumores del músculo liso uterinos pueden presentar patrones de crecimiento anormalmente agresivos, incluyendo la leiomiomatosis intravenosa o peritoneal, metástasis "benignas" y el leiomioma parasítico ${ }^{(71)}$. No existen series en la literatura acerca de esta variante atípica y agresiva de miomatosis, discutiéndose actualmente su real benignidad, en consideración a este patrón de crecimiento, aún cuando microscópicamente no tengan diferencias ${ }^{(72)}$. Teóricamente, el leiomioma parasítico corresponde a una masa tumoral, probablemente subserosa y pedunculada, que en un momento se separó de su origen debido a una torsión sobre el pedículo, adquiriendo nueva vascularización a partir de tejidos adyacentes. Se han descrito distintas ubicaciones intraabdominales, entre ellas peritoneales y mesentéricas. En la TC las características son similares a un leiomioma uterino con significativa vascularización y aspecto nodular o masiforme (Figura 10). Este diagnóstico se puede plantear en presencia de este patrón y el antecedente de un mioma uterino sincrónico o histerectomía previa por miomatosis. No obstante, el mioma parasítico de ubicación mesentérica es fácilmente confundible con procesos malignos que incluyen linfoma, tumor estromal gastrointestinal o carcinoide. La resonancia magnética o el estudio histológico serán necesarios para definir el diagnóstico(71).

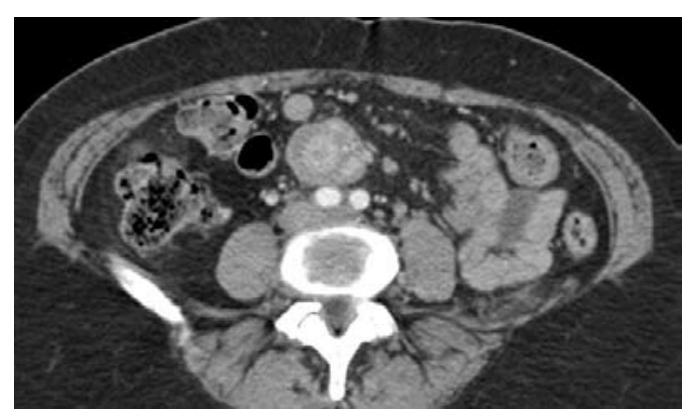

Figura 10. Mioma parasítico. Corte axial de TC: se observa una masa mesentérica bien delimitada, que refuerza con contraste.

\section{Conclusiones}

La mayoría de las masas mesentéricas corresponden a procesos secundarios intraabdominales. El origen primario es poco frecuente, al igual que sus manifestaciones clínicas. La TC es clave para la caracterización de estas lesiones.

\section{Bibliografía}

1. Casillas J, Sais G, Greve J, Iparraguirre M, Morillo G. Imaging of Intra- and Extraabdominal Desmoid Tumors. Radiographics 1992; 11: 959-968.

2. Einstein D, Tagliabue J, Desai R. Abdominal Desmoid: CT findings in 25 patients. Am J Roentgenol 1991; 157: 275-279.

3. Burke A, Thomas R, Elsayed Al, Sobin L. Carcinoids of the jejunum and ileum: an immunohistochemical and clinicopathologic study of 167 cases. Cancer 1997; 79(6): 1086-1093.

4. Kawashima A, Goldman S, Fishman E, Kuhlman J, Onitsuka H, Fukuya T, et al. CT of intraabdominal desmoid tumors: is the tumor different in patients with Gardner's disease? Am J Roentgenol 1994; 162(2): 339-342.

5. Whitley N, Bohlman M, Baker L. CT patterns of mesenteric disease. J Comput Assist Tomogr 1982; 6(3): 490-496.

6. Derchi L, Solbiati L, Rizzatto G, De Pra L. Normal anatomy and pathologic changes of the small bowel mesentery: US appearance. Radiology 1987; 164(3): 649-652.

7. Francis I, Dorovini-Zis K, Glazer G, Lloyd R, Amendola M, Martel W. The fibromatose: CT-pathologic correlation. Am J Roentgenol 1986; 147(5): 1063-1066.

8. Baron R, Lee J. Mesenteric desmoidtumors: sonographic 
and computed-tomographic appearance. Radiology 1981; 140(3): 777-779.

9. Mueller P, Ferrucci J Jr, Harbin W, Kirkpatrick R, Simeone J, Wittenberg J. Appearance of lymphomatous involvement of the mesentery by ultrasonography and body computed tomography: the "sandwich sign". Radiology 1980; 134(2): 467-473.

10. DeMatteo R, Lewis J, Leung D, Mudan S, Woodruff $\mathrm{J}$, Brennan M. Two hundred gastrointestinal stromal tumors: recurrence patterns and prognostic factors for survival. Ann Surg 2000; 231(1): 51-58.

11. Miettinen M, Lasota J. Gastrointestinal stromal tumorsdefinition, clinical, histological, immunohistochemical, and molecular genetic features and differential diagnosis. Virchows Arch 2001; 438(1): 1-12.

12. Emory T, Sobin L, Lukes L, Lee D, O'Leary T. Prognosis of gastrointestinal smooth-muscle (stromal) tumors: dependence on anatomic site. Am J Surg Pathol 1999; 23(1): 82-87.

13. Miettinen M, Monihan J, Sarlomo-Rikala M, Kovatich A, Carr N, Emory T et al. Gastrointestinal stromal tumors/smooth muscle tumors (GISTs) primary in the omentum and mesentery: clinicopathologic and immunohistochemical study of 26 cases. Am J Surg PAthol 1999; 23(9): 1109-1118.

14. Burkill G, Badran M, Al-Muderis O, Meirion Thomas J, Judson I, Fisher C, et al. Malignant gastroinstestinal stromal tumor: distribution, imaging features, and pattern of mestatic spread. Radiology 2003; 226(2): 527-532.

15. Kim H, Lee J, Kim S, Kim K, Lee M, Kim Y, et al. Primary gatrointestinal stromal tumors in the omentum and mesentery: CT findings and pathologic correlations. Am J Roentgenol 2004; 182(6): 1463-1467.

16. Reith J, Goldblum J, Lyles R, Weiss S. Extragastrointestinal (soft tissue) stromal tumosrs: an analysis of 48 cases with emphasis on histologic predictors of outcome. Mod Pathol 2000; 13(5): 577-585.

17. Lee $\mathrm{C}$, Chen $\mathrm{H}$, Leung $\mathrm{T}$, Chen $\mathrm{Y}$. Gastrointestinal stromal tumor: Computed tomographic features. World J Gastroenterol 2004; 10(16): 2417-2418.

18. Levy A, Remotti H, Thompson W, Sobin L, Miettinen M. Gastrointestinal stromal tumors: radiologic features with pathologic correlation. Radiographics 2003; 23(2): 283-304, 456.

19. van Krieken J, Otter R, Hermans J, van Groningen K, Spaander P, van de Sandt M, et al. Malignant lymphoma of the gatrointestinal tract and mesentery. A clinico-pathologic study of the significance of histologic classification. NHL Study Group of the Comprehensive Cancer Center West. Am J Pathol 1989; 135(2): 281289.

20. DiSario J, Burt R, Vargas H, McWhorter W. Small bowel cancer: epidemiological and clinical characteristics from a popilation-based registry. Am J Gastroenterol 1994; 89(5): 699-701.

21. Cunningham J, Aleali R, Aleali M, Brower $S$, Aufses A. Malignant small bowel neoplasms: histopathologic determinants of recurrence and survival. Ann Surg 1997; 225(3): 300-306.

22. Ojha A, Zacherl J, Scheuba C, Jakesz R, Wenzl E. Primary small bowel malignancies: single-center results of three decades. J Clin Gastroenterol 2000; 30(3): 289-293.
23. Godwin J 2nd. Carcinoid tumors. An analysis of 2.837 cases. Cancer 1975; 36(2): 560-569.

24. Buckley J, Fishman E. CT evaluation of small bowel neoplasms: spectrum of disease. Radiographics 1998; 18(2): 379-392.

25. Besa C, O' Brien A, Cruz J. Utilidad de la enteroclisis por tomorafía computada (E-TC) en la detección de tumores de intestino delgado. Rev chil radiol 2008; 14(4): 211-218.

26. Sugimoto E, Lörelius L, Eriksson B, Oberg K. Midgut carcinoid tumours. CT appearance. Acta Radiol 1995; 36(4): 367-371.

27. Picus D, Glazer H, Levitt R, Husband J. Computed tomography of abdominal carcinoid tumors. Am J Roentgenol 1984; 143(3): 581-584.

28. Buck J, Sobin L. Carcinoids of the gastrointestinal tract. Radiographics 1990; 10(6): 1081-1095.

29. Moertel C, Sauer W, Dockerty M, Baggenstoss A. Life history of the carcinoid tumor of the small intestine. Cancer 1961; 14: 901-912.

30. Shebani K, Souba W, Finkelstein D, Stark P, Elgadi $\mathrm{K}$, Tanabe $\mathrm{K}$, et al. Prognosis and survival in patients with gastrointestinal tract carcinoid tumors. Ann Surg 1999; 229(6): 815-821.

31. Pantongrag-Brown L, Buetow P, Carr N, Lichtenstein $\mathrm{J}$, Buck J. Calcification and fibrosis in mesenteric carcinoid tumor: CT findings and pathologic correlation. Am J Roentgenol 1995; 164(2): 387-391.

32. Bancks N, Goldstein H, Dodd G Jr. The roentgenologic spectrum of small intestinal carcinoid tumors. Am J Roentgenol Radium Ther Nucl Med 1975; 12(2): 274280.

33. d'Amore F, Christensen B, Brincker $\mathrm{H}$, Pedersen $\mathrm{N}$, Thorling K, Hastrup J, et al. Clinicopathological features and prognostic factors in extranodal non-Hodgkin lymphomas. Danish LYFO Study Group. Eur J Cancer 1991; 27(10): 1201-1208.

34. Rubesin S, Gilchrist A, Bronner M, Saul S, Herlinger $\mathrm{H}$, Grumbach K, et al. Non-Hodgkin lymphoma of the small intestine. Radiographics 1990; 10(6): 985-998.

35. Freeman C, Berg J, Cutler S. Ocurrence and prognosis of extranodal lymphomas. Cancer 1972; 29(1): 252260.

36. Ha C, Cho M, Allen P, Fuller L, Cabanillas F, Cox J. Primary non-Hodgkin lymphoma of the small bowel. Radiology 1999; 211(1): 183-187.

37. Morton J, Leyland M, Vaughan Hudson G, Vaughan Hudson B, Anderson L, Bennett M, et al. Primary gastrointestinal non-Hodgkin's lymphoma: a review of 175 British National Lymphoma Investigation cases. $\mathrm{Br} \mathrm{J}$ Cancer 1993; 67(4): 776-782.

38. Kadin M, Glatstein E, Dorfman R. Clinicopathologic studies of 117 untreated patients subjected to laparotomy for the staging of Hodgkin's disease. Cancer 1971; 27(6): 1277-1294.

39. Goffinet DR, Castellino RA, Kim H, Dorfman RF, Fuks Z, Rosenberg SA, Nelsen T, Kaplan HS. Staging laparotomies in unselected previously untreated patients with non-Hodgkin's lymphomas. Cancer 1973; 32(3): 672-681.

40. Brady L, Asbell S. Malignant lymphoma of the gastrointestinal tract. Radiology 1980; 137(2): 291-298.

41. Hardy S. The sandwich sign. Radiology 2003; 226(3): 651-652. 
42. Sheth S, Horton K, Garland M, Fishman EK. Mesenteric neoplasms: CT appearances of primary and secondary tumors and differential diagnosis. Radiographics 2003; 23(2): 457-473.

43. Bernardino M, Jing B, Wallace S. Computed tomography diagnosis of mesenteric masses. Am J Roentgenol 1979; 132(1): 33-36.

44. Ros P, Olmsted W, Moser R Jr, Dachman A, Hjermstad $B$, Sobin L. Mesenteric and omental cysts: histologic classification with imaging correlation. Radiology 1987; 164(2): 327-332.

45. Takiff H, Calabria R, Yin L, Stabile B. Mesenteric cysts and intra-abdominal cystic lymphangiomas. Arch Surg 1985; 120(11): 1266-1269.

46. Chung M, Brandt M, St-Vil D, Yazbeck S. Mesenteric cysts in children. J Pediatr Surg 1991; 26(11): 13061608.

47. Namasivayam J, Ziervogel M, Hollman A. Case report: volvulus of a mesentericcyst: an unusual complication diagnosed by CT. Clin Radiol 1992; 46: 211-212.

48. Stoupis C, Ros P, Abbitt P, Burton S, Gauger J. Bubbles in the belly: imaging of cystic mesenteric or omental masses. Radiographics 1994; 14(4): 729-737.

49. Sun C, Tang C, Hill J. Mesenteric lymphangioma. A case report with transmission and scanning electron microscopic studies. Arch Pathol Lab Med. 1980; 104(6): 316-318.

50. Pintér A, Shubert W, Szemlédy F, Göbel P, Schäfer J, Kustos G. Alimentary tract duplications in infants and children. Eur J Pediatr Surg 1992; 2: 8-12.

51. Macpherson R. Gastrointestinal tract duplications: clinical, pathologic, etiologic, and radiologic considerations. Radiographics 1993; 13(5): 1063-1080.

52. Iyer C, Mahour G. Duplications of the alimentary tract in infants and children. J Pediatr Surg 1995; 30(9): 1267-1270.

53. Bowen B, Ros P, McCarthy M, Olmsted W, Hjermstad B. Gastrointestinal teratomas: CT and US appearance with pathologic correlation. Radiology 1987; 162(2): 431-433.

54. Hart J, Mazrani W, Jones N, Kiely E, Sebire N, McHugh K. Upper abdominal teratomas in infants: radiological findings and importance of the vascular anatomy. Pediatr Radiol 2008; 38(7): 750-755.

55. Rose A, Gatto A, Watson J. Recent increases in tuberculosis Recent increases in tuberculosis notifications in England and Wales-real or artefact? J Public Health Med 2002; 24(2): 136-137.

56. Jadvar H, Mindelzun R, Olcott E, Levitt D. Still the great mimicker: abdominal tuberculosis. Am J Roentgenol
1997; 168(6): 1455-1460.

57. Hulnick D, Megibow A, Naidich D, Hilton S, Cho K, Balthazar E. Abdominal tuberculosis: CT evaluation. Radiology 198; 157(1): 199-204.

58. Burrill J, Williams C, Bain G, Conder G, Hine A, Misra $R$. Tuberculosis: a radiologic review. Radiographics 2007; 27(5): 1255-1273.

59. Epstein B, Mann J. CT of abdominal tuberculosis. Am J Roentgenol 1982; 139(5): 861-866.

60. Pickhardt $P$, Bhalla S. Unusual nonneoplastic peritoneal and subperitoneal conditions: CT findings. Radiographics 2005; 25(3): 719-730.

61. Radin D. Intraabdominal Mycobacterium tuberculosis vs Mycobacterium avium-intracellulare infections in patients with AIDS: distinction based on CT findings. Am J Roentgenol 1991; 156(3): 487-491.

62. Hulnick D, Megibow A, Naidich D, Hilton S, Cho K, Balthazar E. Abdominal tuberculosis: CT evaluation. Radiology 1985; 157(1): 199-204.

63. Horton K, Lawler L, Fishman E. CT findings in sclerosing mesenteritis (panniculitis): spectrum of disease. Radiographics 2003; 23(6): 1561-1567.

64. Katz M, Heiken J, Glazer H, Lee J. Intraabdominal panniculitis: clinical, radiographic, and CT features. Am J Roentgenol 1985; 145(2): 293-296.

65. Ogden W 2nd, Bradburn D, Rives J. Mesenteric Panniculitis: Review of 27 Cases. Ann Surg 1965; 161: 864-865.

66. Sabaté J, Torrubia S, Maideu J, Franquet T, Monill J, Pérez C. Sclerosing mesenteritis: imaging findings in 17 patients. Am J Roentgenol 1999; 172(3): 625-629.

67. Healy J, Reznek R. The peritoneum, mesenteries and omenta: normal anatomy and pathological processes. Eur Radiol 1998; 8(6): 886-900.

68. Madureira A. The comb sign. Radiology 2004; $230(3)$ : 783-784.

69. Gore R, Balthazar E, Ghahremani G, Miller F. CT features of ulcerative colitis and Crohn's disease. Am J Roentgenol 1996; 167(1): 3-15.

70. Goldberg H, Gore R, Margulis A, Moss A, Baker E. Computed tomography in the evaluation of Crohn disease. Am J Roentgenol 1983; 140(2): 277-282.

71. Ueda H, Togashi K, Konishi I, Kataoka M, Koyama T, Fujiwara T, et al. Unusual appearances of uterine leiomyomas: MR imaging findings and their hispathologic backgrounds. Radiographics 1999; 19: S131-S145.

72. Cohen D, Oliva E, Hahn P, Fuller A Jr, Lee S. Uterine smooth-muscle tumors with unusual growth patterns: imaging with pathologic correlation. Am J Roentgenol 2007; 188(1): 246-255. 